\title{
Predictive Factors for Pathologic Complete Response Following Neoadjuvant Chemoradiotherapy for Rectal Cancer
}

\author{
Qi Zhang, Jianwei Liang, Jianan Chen, Shiwen Mei, Zheng Wang*
}

\begin{abstract}
Background: An accurate assessment of potential pathologic complete response(pCR) following neoadjuvant chemoradiotherapy(NCRT) is important for the appropriate treatment of rectal cancer. However, the factors that predict the response to neoadjuvant chemoradiotherapy have not been well defined. Therefore, this study analyzed the predictive factors on the development of pCR after neoadjuvant chemoradiation for rectal cancer. Methods: From January 2008 to January 2018, a total of 432 consecutive patients from a single institution patients who underwent a long-course neoadjuvant chemoradiotherapy were reviewed in this study. The clinicopathological features were analyzed to identify predictive factors for pathologic complete response in rectal cancer after neoadjuvant chemoradiation. Results: The rate of pathologic complete response in rectal cancer after neoadjuvant chemoradiation was $20.8 \%$, patients were divided into the $\mathrm{pCR}$ and non-pCR groups. The two groups were well balanced in terms of age, gender, body mass index, ASA score, tumor stage, tumor differentiation, tumor location, surgical procedure, chemotherapy regimen and radiation dose. The multivariate analysis revealed that a pretreatment carcinoembryonic antigen (CEA) level of $\leq 5 \mathrm{ng} /$ $\mathrm{mL}$ and an interval of $\geq 8$ weeks between the completion of chemoradiation and surgical resection were independent risk factors of an increased rate of pCR. Conclusions: Pretreatment carcinoembryonic antigen (CEA) level of $\leq 5 \mathrm{ng} /$ $\mathrm{mL}$ and an interval of $\geq 8$ weeks between the completion of chemoradiation and surgical resection are predictive factors for pathologic complete response in rectal cancer after neoadjuvant chemoradiation. Using these predictive factors, we can predict the prognosis of patients and develop adaptive treatment strategies. A wait-and-see policy might be possible in highly selective cases.
\end{abstract}

Keywords: Rectal cancer- pathologic complete response- neoadjuvant chemoradiotherapy- CEA- interval

Asian Pac J Cancer Prev, 22 (5), 1607-1611

\section{Introduction}

Neoadjuvant chemoradiotherapy (NCRT) and total mesorectal excision (TME) have been shown to significantly decrease the local recurrence rate and improve the overall survival (OS) rate (Sauer et al., 2004; Sauer et al., 2012), which have become the standard of care for patients with clinical stages II and III rectal cancer (Fleming et al., 2011). Neoadjuvant chemoradiotherapy also can increases the rate of sphincter preservation (Glimelius, 2013). Most patients respond to neoadjuvant chemoradiotherapy. After neoadjuvant chemoradiotherapy, about $10 \%-30 \%$ of the patients get complete pathological response, and there is no viable tumor cells in the surgical specimens (Martin et al., 2012). However, some patients did not respond to neoadjuvant chemoradiotherapy. Recent studies have shown that the prognosis of rectal cancer patients with pathological complete response after neoadjuvant chemoradiotherapy is better than that without pathological complete response (Maas et al., 2010a; de Campos-Lobato et al., 2011b). A wait-and-see policy might be a consideration in rectal cancer patients treated with neoadjuvant chemoradiotherapy and who have already clinical complete responded to treatment (Habr-Gama et al., 2004; Maas et al., 2011).

Patients with ypT0N0 rectal cancer are a subgroup that responds well to neoadjuvant chemoradiotherapy and have favorable oncological prognosis, with a 5-year disease-free survival (DFS) rate reaching 83\%-95\% (Stipa et al., 2006; Capirci et al., 2008; Maas et al., 2010b; Zorcolo et al., 2012; Wasmuth et al., 2016). So, the ability to predict the efficacy of neoadjuvant chemoradiotherapy for rectal cancer has important clinical significance. However, it is very difficult to predict the pathological complete response of rectal cancer after neoadjuvant chemoradiotherapy, and the relevant research is limited.

The present retrospective study was designed to evaluate the clinical factors that can be predicted pathologic complete response to neoadjuvant chemoradiotherapy for rectal cancer. 


\section{Materials and Methods}

\section{Patients and methods}

Patients and evaluation before the treatment

This retrospective study was conducted at the Department of Colorectal Surgery, National Cancer Center/National Clinical Research Center for Cancer/Cancer Hospital, Chinese Academy of Medical Sciences and Peking Union Medical College, Beijing, China. The study was performed in accordance with the ethical standards of our institutional research committee, and the principles of the 1964 Declaration of Helsinki and its later amendments. Informed consent was obtained from each individual participant included in the study. The data of 432 consecutive patients with rectal cancer who received neoadjuvant chemoradiotherapy in our hospital from January 2008 to January 2018 were retrospectively analyzed. The criteria for neoadjuvant chemoradiotherapy were as follows: (I) low or middle rectal carcinoma (a distance of $<10 \mathrm{~cm}$ between the inferior tumor edge and the anal verge); (II) pretreatment clinical stage was II and III; (III) no obvious distant metastasis; (IV) postoperative pathological results showed $\mathrm{R} 0$ resection. The exclusion criteria were: (I) other malignant tumors present (except for rectal cancers); (II) a history of malignant tumor or relapse; (III) managed by a watch-and-wait strategy after neoadjuvant chemoradiotherapy.

All patients underwent colonoscopy with biopsy and were histologically diagnosed with adenocarcinoma. Preoperative clinical staging was determined by abdominal and pelvic computed tomography (CT), transrectal ultrasonography, pelvic magnetic resonance imaging, or a combination of these. Postoperative specimens were examined by at least two pathologists specialized in colorectal cancer. The specimens were examined grossly and microscopically. pCR was defined as the absence of viable tumor cells in the surgical specimen, including lymph nodes. Patients without pCR were grouped into the non-pCR cohort. Clinicopathological classification and staging were based on the American Joint Committee on Cancer tumor-node-metastasis (TNM) staging system.

\section{Treatment}

All of the patients received intensity-modulated radiation therapy with concurrent oral administration of capecitabine: the total dosage was 50Gy (2.0 Gy per time, 25 fractions). One of the two chemotherapeutic regimens was delivered concurrently with radiotherapy as follows: oral capecitabine at a dose of $1,650 \mathrm{mg} / \mathrm{m}^{2}$ per day for 35 days, without weekend breaks, or oral capecitabine at a dose of $1,650 \mathrm{mg} / \mathrm{m}^{2}$ per day for 35 days plus intravenous oxaliplatin at a dose of $50 \mathrm{mg} / \mathrm{m}^{2}$ once weekly for 5 weeks. Surgical resection was planned for 6-8weeks after the completion of neoadjuvant chemoradiotherapy, irrespective of the response to chemoradiotherapy. All patients underwent radical total mesorectal excision (TME).

The clinicopathological and operative data were documented, including age, gender, body mass index, ASA score, tumor stage, tumor differentiation, tumor location, surgical procedure, pretreatment serum CEA level, chemotherapy regimen, the interval between neoadjuvant chemoradiotherapy and surgery, and radiation dose. Data of last follow-up and vital status were collected on all patients. Each patient was followed-up every three months for the first two years, every six months for the next three years, and once a year thereafter. Digital rectal examination was performed and the levels of carcinoembryonic antigen (CEA) and carbohydrate antigen (CA) 19-9 were determined at every follow-up visit. Chest computed tomography, magnetic resonance imaging, or computed tomography with intravenous contrast of the liver and pelvis, and full colonoscopy were regularly undertaken.

\section{Statistical analysis}

Nonparametric variables are presented as the median and range, and categorical variables are presented as the frequency with percentages. Continuous variables were analyzed with the Mann-Whitney U test, and categorical variables were analyzed with the Chisquare test or Fisher's exact test, when appropriate. univariate and multivariate analyses were performed by using logistic regression and Cox proportional hazard ratios to identify factors that predict $\mathrm{pCR} . \mathrm{P}<0.05$ was considered statistically significant. Data were analyzed by Statistical Package for the Social Science (SPSS) for Windows (SPSS Inc., Chicago, IL, USA).

\section{Results}

Patient demographics and tumor characteristics are shown in Table 1 . In our hospital, a total of 432 consecutive patients with clinical stages II and III rectal adenocarcinoma who underwent long-course neoadjuvant chemoradiotherapy followed by TME were included in this study. Of the 432 patients, 90 (20.8\%) achieved a pCR, and 342 (79.2\%) did not. The patients were divided into two groups, the pCR $(n=90)$ and non-pCR $(n=342)$ groups. The age, gender, body mass index, ASA score, tumor stage, tumor differentiation, tumor location, surgical procedure, chemotherapy regimen and radiation dose were not statistically different between the two groups. The pretreatment serum CEA level was significantly lower in the pCR group than in the non-pCR group (2.6 vs. $5.8 \mathrm{ng} / \mathrm{mL}, \mathrm{P}=0.001)$. The median interval between the completion of neoadjuvant chemoradiotherapy and surgery was significantly longer in the $\mathrm{pCR}$ group than in the non pCR group (56 vs. 49 days, $\mathrm{P}<0.001$ ).

For the univariate and multivariate analyses, the variables were analyzed as discrete categorical variables. On multivariate analysis, a pretreatment CEA level of $\leq 5 \mathrm{ng} / \mathrm{mL}(\mathrm{OR}=1.9,95 \% \mathrm{CI}=1.22-3.84, \mathrm{P}=0.018)$ and an interval from the completion of neoadjuvant chemoradiotherapy to surgery of $\geq 8$ weeks (OR=2.1, 95\% $\mathrm{CI}=1.81-5.06, \mathrm{P}=0.009)$ were identified as independent predictors for achieving a pCR (Table 2).

\section{Discussion}

For clinical stages II and III rectal cancer, the standard treatment is neoadjuvant chemoradiotherapy and surgery. 
DOI: 10.31557/APJCP.2021.22.5.1607

Pathologic Complete Response Following Neoadjuvant Chemoradiotherapy for Rectal Cancer

Table 1. Patient Demographics and Tumor Characteristics

\begin{tabular}{|c|c|c|c|}
\hline Variable & $\mathrm{pCR}(\mathrm{n}=90)$ & Non-pCR $(n=342)$ & $P$ value \\
\hline Age (years) & $60(26-86)$ & $59(32-82)$ & 0.812 \\
\hline Gender (male / female) & $53 / 37$ & $189 / 153$ & 0.564 \\
\hline $\operatorname{BMI}\left(\mathrm{kg} / \mathrm{m}^{2}\right)$ & $24.6(17.4-33.2)$ & $24.5(17.0-32.8)$ & 0.462 \\
\hline ASA score & & & 0.735 \\
\hline 1 & $11(12.2 \%)$ & $51(14.9 \%)$ & \\
\hline 2 & $46(51.1 \%)$ & $158(46.2 \%)$ & \\
\hline 3 & $33(36.7 \%)$ & $133(38.9 \%)$ & \\
\hline cT category & & & 0.258 \\
\hline 2 & $9(10.0)$ & $30(8.8)$ & \\
\hline 3 & $73(81.1)$ & $256(74.9)$ & \\
\hline 4 & $8(8.9)$ & $56(16.4)$ & \\
\hline $\mathrm{cN}$ category & & & 0.784 \\
\hline No & $26(28.9)$ & $96(28.0)$ & \\
\hline N1 & $44(48.9)$ & $169(49.4)$ & \\
\hline $\mathrm{N} 2$ & $20(22.2)$ & $77(22.5)$ & \\
\hline Tumor stage & & & 0.884 \\
\hline II & $28(31.1)$ & $96(28.0)$ & \\
\hline III & $62(68.9)$ & $181(71.9)$ & \\
\hline Differentiation & & & 0.715 \\
\hline Well & $8(8.9 \%)$ & $28(8.2 \%)$ & \\
\hline Moderately & $71(78.9 \%)$ & $265(77.5 \%)$ & \\
\hline Poor & $11(12.2 \%)$ & $49(14.3 \%)$ & \\
\hline Distance of tumor from the anal verge $(\mathrm{cm})$ & $6(0-10)$ & $6(0-10)$ & 0.845 \\
\hline Pretreatment CEA (ng/mL) & $2.6(0.05-24.9)$ & $5.8(0.02-35.8)$ & 0.001 \\
\hline Surgical procedure & & & 0.539 \\
\hline Anterior resection & $52(57.8 \%)$ & $208(60.8 \%)$ & \\
\hline Abdominoperineal resection & $32(35.6 \%)$ & $120(35.1 \%)$ & \\
\hline Hartmann procedure & $6(6.7 \%)$ & $14(4.1 \%)$ & \\
\hline Chemotherapy & & & 0.712 \\
\hline Capecitabine & $75(83.3)$ & $285(83.3)$ & \\
\hline Capecitabine + oxaliplatin & $15(16.7)$ & $57(16.7)$ & \\
\hline Time interval (day) & $56(35-95)$ & $49(25-105)$ & $<0.001$ \\
\hline Radiation dose (Gy) & $50(40-60)$ & $50(38-60)$ & 0.354 \\
\hline
\end{tabular}

Table 2. Predictors for Pathologic Complete Response by Multivariate Analysis

\begin{tabular}{lccc}
\hline Factor & OR & $95 \%$ CI & P-value \\
\hline Serum CEA $(\mathrm{ng} / \mathrm{mL}) \leq 5$ & 1.9 & $1.22-3.84$ & 0.018 \\
Time interval $($ weeks $) \geq 8$ & 2.1 & $1.81-5.06$ & 0.009 \\
\hline
\end{tabular}

Compared with surgical resection alone or postoperative chemoradiotherapy, neoadjuvant chemoradiotherapy improves local control. The prognosis of patients with tumor regression and $\mathrm{T}$ or $\mathrm{N}$ downstaging after neoadjuvant chemoradiotherapy for rectal cancer has been shown to improve significantly. It has been well documented that patients who achieved pCR had better long-term outcomes than those without pCR. However, the factors that predict patient response to neoadjuvant chemoradiotherapy for rectal cancer have not been well defined. Some studies have evaluated clinical factors associated with complete response to preoperative chemoradiotherapy for rectal cancer (García-Aguilar et al., 2003; Das et al., 2007b; Kalady et al., 2009b; Park et al., 2011; Garland et al., 2014a). Armstrong et al., (2015) found that lower pretreatment CEA level, proximity to anal verge, and statin use were predictors of $\mathrm{pCR}$ after evaluating the clinical factors of 885 patients. Das et al., (2007a) identified that the circumferential tumor extent was the only factor that was significantly associated with pCR in a large sample size retrospective review of 562 patients. Kalady et al., (2009a) evaluated the predictors of pCR in 242 patients and found that an interval of $>8$ weeks between the completion of preoperative chemoradiotherapy and surgical resection was the only factor that was significantly associated with pCR. Garland et al., (2014b) found that the tumor size and pretreatment clinical $\mathrm{N}$ category were independent predictors of $\mathrm{pCR}$ after evaluating the clinical factors of 297 patients. In this 
analysis of a consecutive series of rectal cancer patients who underwent neoadjuvant chemoradiotherapy and radical resection from a single institution, the rate of postoperative pathologic complete response(pCR) was $20.8 \%$, and we found that a pretreatment CEA level of $\leq 5 \mathrm{ng} / \mathrm{mL}$ and an interval from the completion of neoadjuvant chemoradiotherapy to surgery of $\geq 8$ weeks were independent predictors of $\mathrm{pCR}$.

Carcinoembryonic antigen (CEA) is a tumor associated antigen, CEA has important clinical value in the condition monitoring and curative effect evaluation of colorectal cancer (Berman et al., 2000). In colon cancer, CEA levels have been shown to be an important prognostic factor in node-negative disease, with an increase of the preoperative serum CEA level associated with an increased risk of recurrence (Harrison et al., 1997). Consistent with our findings, Yeo et al., (2013) also showed that CEA level before treatment was an important predictive factor of pCR in a cohort of 609 patients who received preoperative chemoradiotherapy. Riesterer et al., (2007) found that tumor cells which have a high density of CEA may resist radiation. Garland et al., (2014c) found the pretreatment serum CEA levels and a decrease in the pre- to post-treatment serum CEA level were independent risk factors of $\mathrm{pCR}$. Another recent study confirmed the value of CEA level before treatment on $\mathrm{pCR}$, but found that the predictive value was limited to patients who were nonsmokers (Wallin et al., 2013). We found that the CEA level was significantly higher in the non-pCR group than in the pCR group, and a normal pretreatment CEA level was significantly associated with pCR in both univariate and multivariate analyses.

In 1999, François et al., (1999) (The Lyon Trial) advocated the adoption of an interval between chemoradiation and surgery of 6 to 8 weeks. This was based on a statistically nonsignificant improvement in sphincter preservation rates without changes in perioperative complications, compared to an interval of 2-3 weeks. Based on these equivocal findings, an interval between chemoradiation and surgery of 6 to 8 weeks has become part of the standard protocol for the treatment of rectal cancer in the USA. Radiation-induced necrosis and subsequent tumor regression is a time-dependent phenomenon in which a longer interval between the completion of chemoradiotherapy and surgery may increase the rate of pCR (Kalady et al., 2009c). Therefore, the persistent effects of neoadjuvant treatment would continue to cause cell death over time, and consequently, waiting longer before surgery could yield less viable carcinoma at the time of surgery. In this study, we found that a time interval between chemoradiation and surgery $\geq 8$ weeks was the independent predictor of $\mathrm{pCR}(\mathrm{OR}=2.1,95 \% \mathrm{CI}=1.81-5.06, \mathrm{P}=0.020)$. Similarly, Kalady et al. reported that an interval of $>8$ weeks was an independent predictor for $\mathrm{pCR}(\mathrm{OR}=2.63,95 \% \mathrm{CI}$ $=1.13-6.12, \mathrm{P}=0.020$ ) (Kalady et al., 2009c). Wolthuis et al., (2012) reported that an interval of $>7$ weeks was associated with increased pCR ( 28 vs. $16 \%, \mathrm{P}=0.030)$, and the 5-year cancer-specific survival rate was higher in the long-interval group than in the short-interval group
(91\% vs. $83 \%, \mathrm{P}=0.046)$.

de Campos-Lobato et al., (2011a) has demonstrated that a prolonged interval between chemoradiation and surgery was an independent predictor of achieving a $\mathrm{pCR}$, and this study shows that a longer interval is safe for patients as it does not increase peri or postoperative morbidity. Furthermore, an interval of greater than or equal to 8 weeks resulted in a decreased rate of local recurrence.

Despite attempts to improve tumor response by varying chemotherapy regimens, no significant impact on oncologic outcomes has been made. To improve the response, some randomized trials have added oxaliplatin or targeted drugs into the currently widely used fluorinated, pyrimidine-based preoperative chemotherapy regimen (Gérard et al., 2012; O'Connell et al., 2014). However, none of these studies reported an increased pCR rate. Our study found that the addition of oxaliplatin did not increase the $\mathrm{pCR}$ rate.

Due to the retrospective nature of this study, it is subject to potential bias and certain limitations. Firstly, this is a single-center, retrospective study. Besides, there were fewer patients in the pCR group than in the non-pCR group, there may be bias. Secondly, Since the study was not prospectively designed, the time interval between chemoradiation and surgery was decided according to the individual surgeon preference. To solve these controversial factors, randomized clinical trials should be performed in the future and identify the predictors for pCR and elucidate its potential mechanisms. Patients who are considered good responders to neoadjuvant chemoradiotherapy may benefit little from TME, and their optimal treatment may involve nonoperative management or local excision.

In conclusion, this large, retrospective study demonstrated that a pretreatment CEA level of $\leq 5 \mathrm{ng} /$ $\mathrm{mL}$ and an interval from the completion of neoadjuvant chemoradiotherapy to surgery of $\geq 8$ weeks were independent clinical predictors for achieving pCR. These findings may help clinicians predict the prognosis of patients and develop individualized treatment strategies.

\section{Author Contribution Statement}

Zheng Wang and Qi Zhang contributed the conceiving and designing the study. Jianwei Liang and Jianan Chen did the collecting of data. Shiwen Mei did the analyzing and interpreting of data. Qi Zhang and Zheng Wang did the writing of the article. Zheng Wang approved the final version of the article.

\section{Acknowledgments}

\section{Conflict of interest statement}

We declare that we have no financial and personal relationships with other people or organizations that can inappropriately influence our work, there is no professional or other personal interest of any nature or kind in any product, service and/or company that could be construed as influencing the position presented in, or the 
review of the manuscript entitled. The paper is not based on a previous communication to a society or meeting. The paper has gained ethics committee approval.

\section{References}

Armstrong D, Raissouni S, Price Hiller J, et al (2015). Predictors of pathologic complete response after neoadjuvant treatment for rectal cancer: A Multicenter Study. Clin Colorectal Cancer, 14, 291-5.

Berman JM, Cheung RJ, Weinberg DS (2000). Surveillance after colorectal cancer resection. Lancet, 355, 395-9.

Capirci C, Valentini V, Cionini L, et al (2008). Prognostic value of pathologic complete response after neoadjuvant therapy in locally advanced rectal cancer: long-term analysis of 566 ypCR patients. Int J Radiat Oncol Biol Phys, 72, 99-107.

Das P, Skibber JM, Rodriguez-Bigas MA, et al (2007a). Predictors of tumor response and downstaging in patients who receive preoperative chemoradiation for rectal cancer. Cancer, 109, 1750-5.

de Campos-Lobato LF, Geisler DP, da Luz Moreira A, et al (2011a). Neoadjuvant therapy for rectal cancer: the impact of longer interval between chemoradiation and surgery. J Gastrointest Surg, 15, 444-50.

Fleming FJ, Påhlman L, Monson JR (2011). Neoadjuvant therapy in rectal cancer. Dis Colon Rectum, 54, 901-12.

Francois Y, Nemoz CJ, Baulieux J, et al (1999). Influence of the interval between preoperative radiation therapy and surgery on downstaging and on the rate of sphincter-sparing surgery for rectal cancer: the Lyon R90-01 randomized trial. J Clin Oncol, 17, 2396.

Gérard JP, Azria D, Gourgou-Bourgade S, et al (2012). Clinical outcome of the ACCORD 12/0405 PRODIGE 2 randomized trial in rectal cancer. J Clin Oncol, 30, 4558-65.

García-Aguilar J, Hernandez de Anda E, Sirivongs P, et al (2003). A pathologic complete response to preoperative chemoradiation is associated with lower local recurrence and improved survival in rectal cancer patients treated by mesorectal excision. Dis Colon Rectum, 46, 298-304.

Garland ML, Vather R, Bunkley N, et al (2014a). Clinical tumour size and nodal status predict pathologic complete response following neoadjuvant chemoradiotherapy for rectal cancer. Int J Colorectal Dis, 29, 301-7.

Glimelius B (2013). Neo-adjuvant radiotherapy in rectal cancer. World J Gastroenterol, 19, 8489-501.

Habr-Gama A, Perez RO, Nadalin W, et al (2004). Operative versus nonoperative treatment for stage 0 distal rectal cancer following chemoradiation therapy: long-term results. Ann Surg, 240, 711-7; discussion 7-8.

Harrison LE, Guillem JG, Paty P, et al (1997). Preoperative carcinoembryonic antigen predicts outcomes in node-negative colon cancer patients: a multivariate analysis of 572 patients. $J$ Am Coll Surg, 185, 55-9.

Kalady MF, de Campos-Lobato LF, Stocchi L, et al (2009a). Predictive factors of pathologic complete response after neoadjuvant chemoradiation for rectal cancer. Ann Surg, 250, 582-9.

Maas M, Beets-Tan RG, Lambregts DM, et al (2011). Wait-and-see policy for clinical complete responders after chemoradiation for rectal cancer. J Clin Oncol, 29, 4633-40.

Maas M, Nelemans PJ, Valentini V, et al (2010a). Long-term outcome in patients with a pathological complete response after chemoradiation for rectal cancer: a pooled analysis of individual patient data. Lancet Oncol, 11, 835-44.

Martin ST, Heneghan HM, Winter DC (2012). Systematic review and meta-analysis of outcomes following pathological complete response to neoadjuvant chemoradiotherapy for rectal cancer. Br J Surg, 99, 918-28.

O'Connell MJ, Colangelo LH, Beart RW, et al (2014). Capecitabine and oxaliplatin in the preoperative multimodality treatment of rectal cancer: surgical end points from National Surgical Adjuvant Breast and Bowel Project trial R-04. J Clin Oncol, 32, 1927-34.

Park CH, Kim HC, Cho YB, et al (2011). Predicting tumor response after preoperative chemoradiation using clinical parameters in rectal cancer. World J Gastroenterol, 17, 5310-6.

Riesterer O, Milas L, Ang KK (2007). Use of molecular biomarkers for predicting the response to radiotherapy with or without chemotherapy. J Clin Oncol, 25, 4075-83.

Sauer R, Becker H, Hohenberger W, et al (2004). Preoperative versus postoperative chemoradiotherapy for rectal cancer. N Engl J Med, 351, 1731-40.

Sauer R, Liersch T, Merkel S, et al (2012). Preoperative versus postoperative chemoradiotherapy for locally advanced rectal cancer: results of the German CAO/ARO/AIO-94 randomized phase III trial after a median follow-up of 11 years. J Clin Oncol, 30, 1926-33.

Stipa F, Chessin DB, Shia J, et al (2006). A pathologic complete response of rectal cancer to preoperative combined-modality therapy results in improved oncological outcome compared with those who achieve no downstaging on the basis of preoperative endorectal ultrasonography. Ann Surg Oncol, 13, 1047-53.

Wallin U, Rothenberger D, Lowry A, et al (2013). CEA - a predictor for pathologic complete response after neoadjuvant therapy for rectal cancer. Dis Colon Rectum, 56, 859-68.

Wasmuth HH, Rekstad LC, Tranø G (2016). The outcome and the frequency of pathological complete response after neoadjuvant radiotherapy in curative resections for advanced rectal cancer: a population-based study. Colorectal Dis, $\mathbf{1 8}$, 67-72.

Wolthuis AM, Penninckx F, Haustermans K, et al (2012). Impact of interval between neoadjuvant chemoradiotherapy and TME for locally advanced rectal cancer on pathologic response and oncologic outcome. Ann Surg Oncol, 19, 2833-41.

Yeo SG, Kim DY, Chang HJ, et al (2013). Reappraisal of pretreatment carcinoembryonic antigen in patients with rectal cancer receiving preoperative chemoradiotherapy. Tumori, 99, 93-9.

Zorcolo L, Rosman AS, Restivo A, et al (2012). Complete pathologic response after combined modality treatment for rectal cancer and long-term survival: a meta-analysis. Ann Surg Oncol, 19, 2822-32.

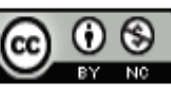

This work is licensed under a Creative Commons AttributionNon Commercial 4.0 International License. 